2. 2021 ESC Guidelines on cardiovascular disease prevention in clinical practice European Heart Journal, Volume 42, Issue 34, 7 September 2021, Pages 3227-3337, https://doi.org/10.1093/eurheartj/ehab484

DOI https://doi.org/10.30525/978-9934-26-182-4-49

\title{
REPRODUCTIVE HEALTH IN THE CONTEXT OF PANDEMIC COVID-19
}

\author{
Nykula B. T. \\ $M D$, \\ Kyiv Clinical Hospital on Railways Transport № 2 \\ Health Centre of JSC "Ukrainian railways"
}

\section{Omelchenko E. M.}

$P h D$,

Head of the Laboratory of genetic epidemiology

State Institution «O.M. Marzelev Institute for Public Health

of the National Academy of Medical Science of Ukraine»

\section{Yelizarova O. T.}

$M D, P h D$,

Leading researcher at the Department of Public Health

State Institution «O.M. Marzelev Institute for Public Health

of the National Academy of Medical Science of Ukraine»

\section{Kyiv, Ukraine}

The onset of the COVID-19 pandemic triggered changes in both the public and private lives of millions of people and triggered negative changes in the health of the population [1]. Studying the consequences of the pandemic and the impact of its various aspects on reproductive health is one of the issues in the focus of attention of scientists.

Reproductive healthcare is a pressing issue for both the health sector and society as a whole [2]. The social significance of reproductive health is based on the increased probability of population decline and age structure change due to fertility rates decreasing in the European region, the United States, Japan, Hong Kong and other developed countries [3]. These changes might have profound economic, social, and geopolitical impacts [3]. 
The reproductive system function is normal oogenesis, spermatogenesis, pregnancy and birth of a healthy child. Necessary conditions of reproductive function are the initiation of the processes of oogenesis and spermatogenesis by secretion of Gonadotropin-Releasing hormone, a satisfactory blood supply to all organs and systems, mental health, as well as adequate metabolic reactions carried out by such hormones as leptin, insulin and ghrelin [4]. Violations at any stage of this process lead to significant and, sometimes, irreversible consequences. Reproductive health is also associated with general and sexual health [3].

An increase in the number of scientific studies of reproductive health is due to various consequences of the development of new coronavirus infection, from psychological to physiological, which carries certain risks of increasing the burden of reproductive losses [2]. Therefore, the aim of this study is to highlight reproductive health research in relation to the COVID-19 pandemic.

Materials and methods. Literature research on the possible mechanisms of reproductive disorders and infertility in the pandemic COVID-19 was performed. Searches were performed in the PubMed, Cochrane Library and Google Scholar databases for the keywords «Reproductive health», «Reproductive disorders», «Erectile dysfunction», «Sexual dysfunction», «Vaccine», «Effectiveness», «COVID-19», «SARS-CoV-2».

Results. As part of the strategy to contain the spread of the new coronavirus infection at the beginning of the pandemic, reproductive care centers in many countries of the world were closed, which led to a number of catastrophic consequences for both men and women, especially in countries such as India and Nepal [5] The containment strategy for this disease has allowed such centers to reopen with certain conditions, but these conditions do not allow the use of reproductive technologies as widely as in the pre-pandemic period. This problem requires an early solution to return the previous volume of support to the population.

The knowledge that the SARS-CoV-2 virus enters the body through the Angiotensin-converting enzyme 2 (ACE2) receptors, which are also found in the organs of the reproductive system, led to the studies association between reproductive disorders as well as pandemic consequences.

Review [6] the mechanisms of possible damage to the reproductive system in men post-COVID-19 due to overexpression of ACE2 receptors, which play a key role in spermatogenesis and in the regulation of steroidogenesis, have been disclosed.

It has also been proven that the risk of developing erectile dysfunction is due to both the direct effect of the virus and damage to the vascular endothelium and other cardiovascular effects. Patients with COVID-19 were 3.3 times more 
likely to have erectile dysfunction with $95 \%$ CI $(2.8,3.8)$ [7]. However, the question of the presence of the SARS-CoV-2 virus in semen and the effect of this fact on fertility is still controversial and requires further research. This is especially true for people with asymptomatic coronavirus infection.

However, it is not only men who are at risk of reproductive harm in relation to the consequences of SARS infection. The study [8] demonstrated that the SARS-CoV-2 infection adversely affects the follicular microenvironment, thus dysregulating ovarian function which possibly increases the probability of reproductive disorders in the future. Other studies [9] have found that SARS-CoV-2, antibody, and other factors, such as the decline of lymphocyte counts, and increased erythrocyte sedimentation rate, C-reactive protein, and D-dimer levels, are evidence of SARS-CoV-2 vertical transmission during pregnancy.

Considering that antibodies against SARS-CoV-2 show different expressions in male and female patients and the issue of vertical transmission of both the virus itself and antibodies during pregnancy, the researchers also look at the consequences of vaccination on the reproductive health of women [2]. The first vaccine trial in pregnant women started in February 2021 (ClinicalTrials.gov identifier NCT04754594) [6]. It is an ongoing phase II/III randomized, placebocontrolled, observer-blinded study of 700 healthy pregnant women, 18 years of age or older, vaccinated during 24-34 weeks of gestation. The study will evaluate safety, tolerability and immunogenicity of two doses of Comirnaty vs placebo, administered 21 days apart. The study will also assess the transfer of potentially protective antibodies to infants, who will be monitored for safety through approximately 6 months of age.

According to retrospective data, among 3958 pregnancies that were injected with vaccines with COVID-19 mRNA, 115 (13.9\%) ended in miscarriage, which is consistent with population studies before the pandemic [9]. According to the American College of Obstetricians and Gynecologists, in the general population, the incidence of clinically recognized early pregnancy loss in women aged 20 to 30 is $9-17 \%$, and it rises sharply with increasing age (up to $80 \%$ at 45$)$.

Discussing about the consequences of the pandemic for reproductive health, it is impossible to ignore the influence of the psychological factor. Social isolation, the risk of transmission of the virus by direct contact, and hypogonadal state after a previous illness, all of these factors can aggravate pre-existing subclinical sexual dysfunctions [2]. Some studies have shown that pregnant women during a pandemic experience more stress and anxiety than before the pandemic. This can lead to the development of PTSD, especially if they have 
previously experienced anxiety or depressive disorders [9]. This cannot but affect the mental health of infants and young children [10].

Summing up the above, it should be noted that the solution to the problem of preserving reproductive health in the context of the COVID-19 pandemic cannot be implemented without the use of new approaches and the work of a multidisciplinary team of doctors. We can state that the solution to this problem is possible with the use of new technologies and the support of creative industries only.

Conclusions. The pandemic COVID-19 might be a course of sexual and reproductive health issues due to psychological and functional disorders. Further research into these effects is needed at both the individual and population levels.

\section{References:}

1. Building health systems resilience for universal health coverage and health security during the COVID-19 pandemic and beyond: a brief on the WHO position. Geneva: World Health Organization; 2021 (WHO/UHL/PHCSP/2021.02). https://apps.who.int/iris/bitstream/handle/10665/346515/WHO-UHL-PHC-SP2021.01-rus.pdf

2. Liu C., Mu C., Zhang Q. et al. Effects of Infection with SARS-CoV-2 on the Male and Female Reproductive Systems: A Review. Med Sci Monit. 2021. Vol. 27. e930168. doi: 10.12659/MSM.930168.

3. Vollset S.E., Goren E., Yuan C.W. et al. Fertility, mortality, migration, and population scenarios for 195 countries and territories from 2017 to 2100: a forecasting analysis for the Global Burden of Disease Study. Lancet. 2020. Vol. 396. P.1285-1306. doi: 10.1016/S0140-6736(20)30677-2.

4. Evans M.C., Anderson G.M. Neuroendocrine integration of nutritional signals on reproduction. $J$ Mol Endocrinol. 2017. Vol.58(2). P.107-128. doi: 10.1530/JME-16-0212.

5. Cousins S. COVID-19 has "devastating" effect on women and girls. Lancet. 2020 Aug 1;396(10247):301-302. doi: 10.1016/S0140-6736(20) 31679-2.

6. Sansone A., Mollaioli D., Ciocca G. et al., Addressing male sexual and reproductive health in the wake of COVID-19 outbreak. J Endocrinol Invest. 2021. Vol. 44(2). P.223-231. doi: 10.1007/s40618-020-01350-1.

7. Katz J., Yue S., Xue W. et al. Increased odds ratio for erectile dysfunction in COVID-19 patients. J Endocrinol Invest. 2021. Vol.1. P. 1-6. doi: 10.1007/s40618-021-01717-y. 
8. Herrero Y., Pascuali N., Velázquez C. et al. SARS-CoV-2 infection negatively affects ovarian function in ART patients. Biochim Biophys Acta Mol Basis Dis. 2021. Vol.1. e166295. doi: 10.1016/j.bbadis.2021.166295.

9. Luxi N., Giovanazzi A., Capuano A. et al. COVID-19 Vaccination in Pregnancy, Paediatrics, Immunocompromised Patients, and Persons with History of Allergy or Prior SARS-CoV-2 Infection: Overview of Current Recommendations and Pre- and Post-Marketing Evidence for Vaccine Efficacy and Safety. Drug Saf. 2021. Vol.12. 1247-1269. doi: 10.1007/s40264-02101131-6.

10. Goodman J.H. Perinatal depression and infant mental health. Arch Psychiatr Nurs. 2019. Vol.33. P.217-224. doi: 10.1016/j.apnu.2019.01.010.

DOI https://doi.org/10.30525/978-9934-26-182-4-50

\title{
LEVEL OF PHYSICAL ACTIVITY OF PATIENTS WITH DEPRESSIVE DISORDERS IN THE RESIDUAL PERIOD OF THE DISEASE
}

\author{
Sakharuk L. Yu. \\ Graduate Student at the Department of Physical Therapy \\ and Occupational Therapy \\ Lesya Ukrainka Volyn National University, \\ Lutsk, Ukraine
}

Introduction. Moderate or high physical activity may help reduce the incidence of depressive disorders compared to people with low physical activity. Insufficient physical activity is a risk factor for complications of depressive disorder [1-4].

Aim. To investigate the state of physical activity of patients with depressive disorders in the residual period of the disease.

Materials and methods. Awareness of risk factors and commitment to the prevention of depressive disorders by means of physical activity were assessed based on questionnaires. The survey involved 82 patients with depressive disorders at the stage of outpatient rehabilitation. Of these, 35 were men $(42.41 \%)$ and $47(57.59 \%)$ were women. The average age of men was $60.4 \pm$ 8.1 years, women $-62.2 \pm 8.6$ years.

Results. To better understand the methodology of physical therapy in the residual period of rehabilitation and planning of secondary prevention measures, 184 